\title{
The use of carbon fiber cages in anterior cervical interbody fusion
}

\author{
Report of 100 cases
}

\author{
Khalil Salame, M.D., Georges E. R. Ouaknine, M.D., Nissim Razon, M.D., \\ AND SEMION ROCHKIND, M.D.
}

Department of Neurosurgery, Tel Aviv Sourasky Medical Center, Sackler Faculty of Medicine, Tel Aviv University, Tel Aviv, Israel

\begin{abstract}
Object. Cage devices were introduced in spinal fusion to overcome the shortcomings of autograft, allograft, and biocompatible implants. The aim of this study was to assess the short-term results of anterior cervical discectomy and fusion (ACDF) in which an interbody carbon fiber cage (CFC) and local osteophyte-derived bone graft were implanted.

Methods. A retrospective review was conducted of 100 consecutive patients treated by ACDF in which a CFC was packed with bone fragments obtained from osteophytes at the surgical site. Plain radiographs with dynamic lateral views obtained 1 year postoperatively were used to assess bone fusion, alignment of the cervical spine, and stability. Dynamic radiographs were also obtained at last follow up to determine whether loss of cervical alignment or collapse at the fused disc had occurred.

The mean follow-up period was 25 months. In all cases the cervical lordosis was maintained or corrected to different extents and disc height was restored. Solid fusion was achieved in $98 \%$ of the cases. There were no cage-related complications and no cases of cage failure.

Conclusions. The authors conclude that application of the CFC for ACDF is safe, effective, and technically feasible. Osteophytes resected during surgery may be a good alternative material for bone grafting in cage-assisted cervical interbody fusion.
\end{abstract}

\begin{abstract}
KEY WORDS - anterior cervical interbody fusion - carbon fiber cage - anterior cervical discectomy
\end{abstract}
Since their first application in the cervical spine of a horse, interbody fusion cages have become increasingly popular in spine surgery. The hope has been that interbody fusion cages would help correct structural deformity and maintain stabilization until solid arthrodesis occurs.

The tricortical iliac bone autograft, which has been considered the gold standard for cervical interbody fusion, may be associated with various complications. In one study 15 of the 16 complications occurring after ACDF were graft related. ${ }^{23}$ Sawin, et al. ${ }^{17}$ reported the incidence of donor-site morbidity to be $25.3 \%$ in 300 patients after the iliac crest was harvested for cervical fusion. Donorsite complications may include chronic pain, hematoma, infection, iliac fracture, injury of adjacent nerves, visceral herniation, ureteral injury, and arteriovenous fistula..$^{2,6,8,25}$

In an effort to overcome the shortcomings of autograft, surgeons attempted to use alternative materials such as allografts from bone bank, ${ }^{27}$ methylmethacrylate, ${ }^{9}$ hydroxyapatite, ${ }^{14}$ calcium phosphate derivatives, ${ }^{21}$ and other synthetic biocompatible implants. ${ }^{11}$ These substitutes, like autografts, may be associated with complications at the

\footnotetext{
Abbreviations used in this paper: $\mathrm{ACD}=$ anterior cervical discectomy; $\mathrm{ACDF}=\mathrm{ACF}$ and fusion; $\mathrm{CFC}=$ carbon fiber cage; $\mathrm{MR}=$ magnetic resonance; $\mathrm{VB}=$ vertebral body.
}

implantation site, such as displacement, breakage, or resorption. Another allograft-related risk, albeit a very low one, is the transmission of serious infectious diseases. For a few years, we performed ACDF by using a prosthetic graft (biocompatible osteoconductive polymer). Our experience, like that of others, ${ }^{11}$ has shown that the use of this product was associated with unacceptable rates of implant collapse and fusion failure.

Although the popularity of cervical fusion cages continues to increase, reports on their clinical application remain uncommon. In a review of the literature we found only two published clinical studies on the use of the CFC for cervical interbody fusion. ${ }^{1,3}$ In each of these studies the authors reported on a relatively small number of patients, and the first study ${ }^{1}$ focused only on radiographically demonstrated anatomical results without reporting clinical data. In the present report we summarize our short-term experience of performing $\mathrm{ACDF}$ in which $\mathrm{CFCs}$ were applied in 100 consecutive patients.

\section{CLINICAL MATERIAL AND METHODS}

This retrospective study was based on a review of the hospital charts, operative notes, and out-patient clinical and radiographic follow-up data. 


\section{Interbody $\mathrm{CFC}$}

The CFC (cervical I/F cage; Acromed, Rotterdam, The Netherlands) is a composite of long carbon fibers and a polymer matrix (polyetheretherketone), designed in the shape of a trapezoidal hollow box (Fig. 1). The upper and lower surfaces of the cage are open to allow packing with bone graft and to provide a wide area of contact between the graft and the adjacent VBs, whereas the anterior, posterior and both lateral surfaces are closed to prevent extrusion of the graft. The anterior surface is slightly higher than the posterior, creating a slope of $7^{\circ}$ to preserve cervical lordosis. All surfaces have struts to facilitate weight bearing and ridges to resist displacement. The cage is radiolucent, with one radiopaque tantalum bead incorporated at each of three of its corners (Fig. 2). The construct is available in three different heights $(6,7$, and $8 \mathrm{~mm})$ and two sizes (standard and large).

\section{Surgical Technique}

A standard anterior microdiscectomy is performed under slight distraction created using Caspar posts (Aesculap, Tuttlingen, Germany). The disc, osteophytes, and adjacent cartilaginous endplates are removed. Bone fragments obtained during resection of osteophytes (as well as bone dust acquired during drilling) are collected for grafting. The size and height of the suitable cage is determined using a series of templates. The cage is packed with the bone graft and implanted into the disc space. The posts are removed, leaving the cage under compression. A lateral plain radiograph is obtained to check the position of the cage and the alignment of the cervical spine.

\section{Postoperative Orthosis}

All the patients in this series wore a rigid collar for 1 month after surgery. Lateral radiography was then repeated, and the collar was removed after confirmation of proper location of the cage and good cervical alignment.

\section{Patient Population}

This study included 100 consecutive cases in which ACDF was performed using the CFC. There were 56 men

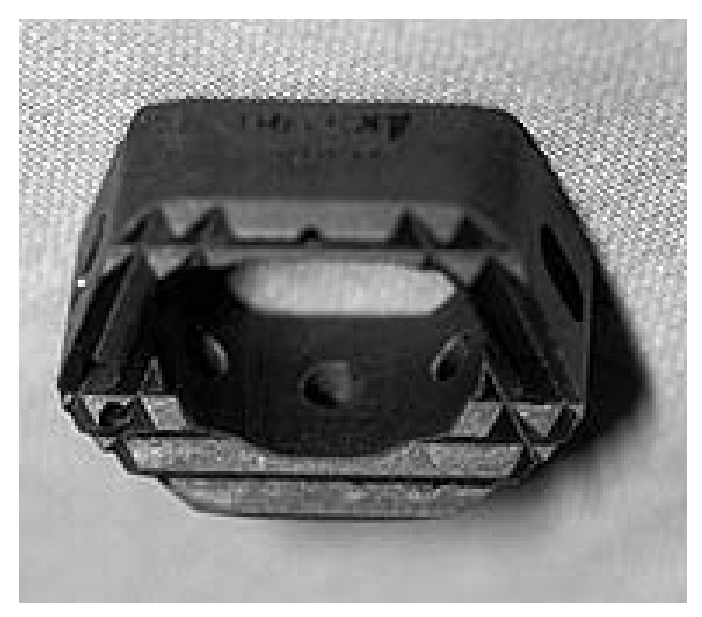

Fig. 1. Photograph showing the CFC.

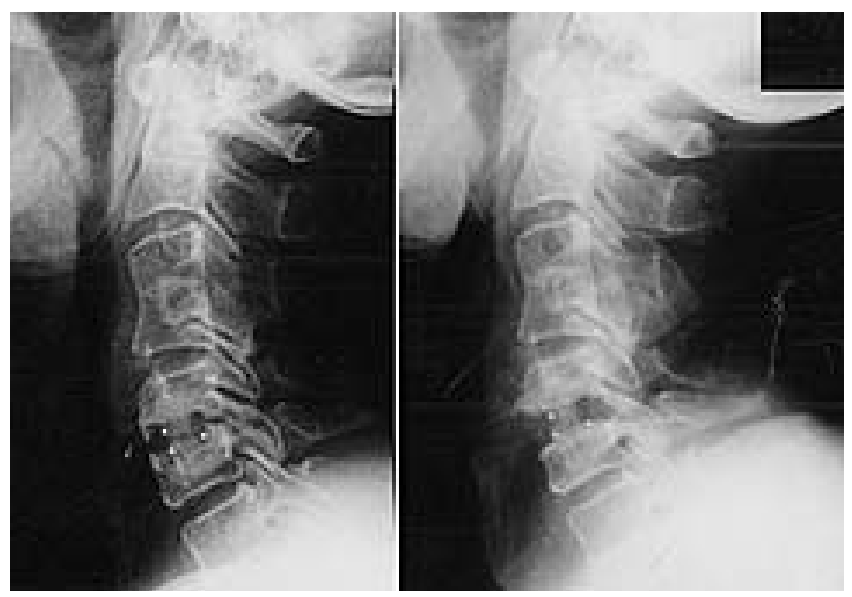

Fig. 2. Lateral radiographs of the cervical spine obtained after $\mathrm{ACDF}$ in which a CFC was placed at C5-6. Although the cage is not visualized, its location is clearly indicated by three radiopaque beads. Left: Six months after surgery, there is a gap at the middle of the bone bridge inside the cage, indicating that bone grows from the endplates into the cage. Osteophytes are shown posterior to the cage. Right: One year postoperatively, bone fusion has occurred and filled the gap. Note resorption of the osteophytes with successful fusion. (Congenital fusion is seen at C3-4).

and 44 women who ranged in age from 19 to 82 years (mean 49 years). Eighty-eight patients suffered from cervical spondylosis and 12 from soft-disc herniation. Fiftythree patients presented with pure radiculopathy without myelopathy, 30 patients with myelopathy without radiculopathy, and 17 with myeloradiculopathy. Forty-one patients also complained of neck pain. Sphincter disturbances were present in 11 patients and sexual disturbances in nine. The peroperative duration of symptoms ranged from 5 days to 42 months (mean 18 months). In 17 patients a history of mild cervical injury was noted. Fortyfive patients were cigarette smokers.

Four patients had previously undergone anterior cervical spinal surgery. In three of them a single-level discectomy was performed without fusion; reoperation and implantion of the CFC were conducted at the same level in two patients and at a different level in the third. One patient had undergone a two-level discectomy and interbody fusion in which an allograft was used 13 months before the present surgery. He presented with progressive quadriparesis and kyphotic deformity related to failed fusion at the caudal level and underwent ACDF in which a CFC was implanted at this level.

Patients in whom additional hardware was implanted concomitantly with the CFC were excluded from this study. This group included: 1) three patients who underwent two-level ACDF in which a CFC was implanted at one level and a threaded titanium cage at the other (these patients were treated earlier in our experience with cages, when we endeavored to determine which cage to use); 2) four patients in whom the CFC was augmented by an anterior plate system (three patients suffered from acute soft-disc herniation associated with ligamentous damage, caused by flexion-distraction injury; one patient had previously undergone a cervical laminectomy); and 3) two patients who underwent one-level discectomy and discec- 
tomy with corpectomy at a nonadjacent level (in these patients we placed a CFC at the discectomy level, a cage designed for VB replacement at the corpectomy/discectomy level, and an anterior plate construct).

\section{Neuroimaging Assessment}

Preoperative investigation included MR imaging in all cases. Preoperatively, cervical lordosis was preserved in 67 patients. Postoperative lateral cervical radiography was performed in all patients on the day after surgery and was repeated at 1 month, 3 months, and 1 year. Fusion was assessed on dynamic lateral radiography obtained 1 year after surgery. Fusion was considered successful if radiography demonstrated trabecular bone bridging between the two VBs and flexion-extension views revealed no movement at the VB (translation) or at the spinous processes. At the time this report was written, dynamic radiographs were also obtained to ensure maintenance of cervical alignment and the height of the disc space at the fused level. To assess alignment, lateral radiographs obtained at the most recent follow-up examinations were compared with similar views acquired in the immediate postoperative period, by laying one film over the other. The height of the disc space was measured on the neutral lateral cervical radiograph.

\section{RESULTS}

The follow-up period ranged from 12 to 40 months (mean 25 months). Surgery was performed at a single vertebral level in 57 patients and at two levels in 43 patients. A total of 143 cages, distributed as follows, were implanted: 15 at C3-4, 42 at C4-5, 60 at C5-6, and 26 at C6-7. The cage size was standard in 132 and large in 11 patients, and the height was $6 \mathrm{~mm}$ in $114,7 \mathrm{~mm}$ in 25 , and $8 \mathrm{~mm}$ in four cases. In all cases the cage was packed with osteophyte bone fragments, thus avoiding the harvesting of a graft from the iliac or any other bone.

\section{Clinical Results}

Of the 41 patients with preoperative neck pain, 27 patients experienced complete pain relief. Significant relief occurred in the remaining 14 patients - the residual pain did not require regular medical treatment. Radicular signs present preoperatively in 70 patients were completely resolved in 61 and improved in nine patients. Of the 47 patients with myelopathy, complete recovery was demonstrated in 13, significant improvement in 21, partial improvement in 10, and no improvement in three, although the progression of myelopathy was halted.

After surgery, two patients required posterior cervical decompression at 19 and 23 months, respectively, because of posterior cord compression with recurrence of myelopathic symptoms. One patient developed adjacent-level discopathy that required surgery 16 months after the first procedure.

\section{Radiographic Results}

After cage implantation, the height of the disc space was maintained or restored in every patient. There was no case of symptomatic cage subsidence, and the mean subsidence at 1 year postoperatively was $1.04 \mathrm{~mm}$. In all 67 patients in whom cervical lordosis was normal preoperatively, it was preserved throughout the follow-up period. Of 33 patients with preoperative loss of physiological lordosis (straight neck, swan neck, or kyphotic deformity) complete correction with restoration of the lordosis was achieved in 18 and partial correction in 15 patients. There were no cases of loss in which cervical alignment occurred during the follow-up period.

Serial radiographs revealed that bone grew into the disc space from the adjacent endplates rather than from the graft within the cage (Fig. 2). Bone also grew around the cage, which became integrated in the new bone (Fig. 3). Solid fusion was achieved in 98 patients. In the other two patients pseudarthrosis was indicated by radiographic lucency at the cage-VB interface, but they were asymptomatic and flexion-extension radiographs demonstrated no movement.

There was no incidence of displacement, breakage or any other cage-related complications, and no cage had to be removed.

\section{Surgery-Related Complications}

Two patients developed postoperative posterior column symptoms (disturbances in proprioception, light touch, and vibration sensibility). They experienced no other sensory or motor disturbances and their symptoms disappeared within 1 month. Three patients suffered from postoperative paresis of the recurrent laryngeal nerve, which resolved spontaneously in two patients within 2 months but persisted in the third patient and required surgical laryngeal reconstruction. In one patient who suffered from dysphagia for several weeks, the results of an upper gastrointestinal barium series and esophagoscopy were unremarkable. One patient developed a wound infection and was treated successfully with antibiotic medication.

\section{DISCUSSION}

Whether interbody fusion is necessary after ACD remains controversial. We believe that successful fusion

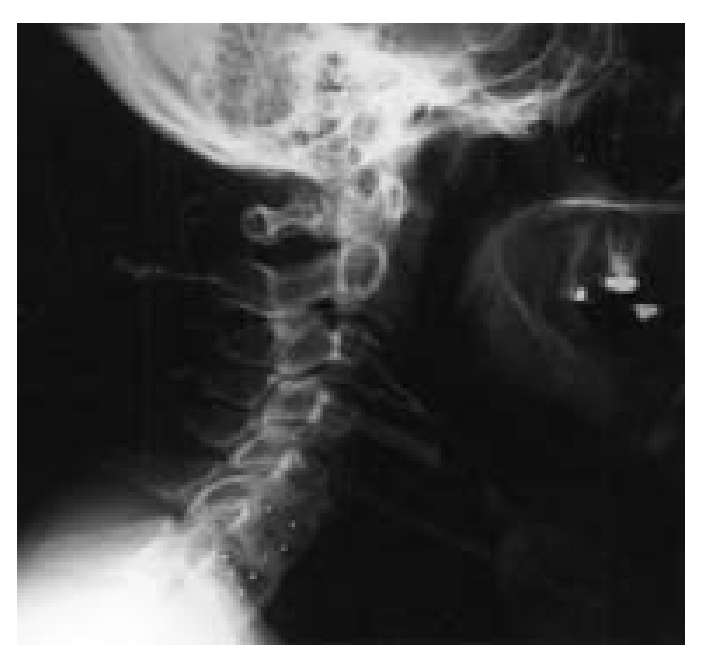

Fig. 3. Lateral cervical radiograph obtained after ACDF in which a CFC was placed at C5-6 and C6-7, demonstrating bone growth inside and around the cage. 
improves the surgery-related outcome in these patients. Caspar and Pitzen ${ }^{4}$ have stated that ACD causes a significant decrease in cervical spinal stiffness. The role of the intervertebral disc in providing cervical stability was also shown in a finite element model reported by Goel and Clausen. ${ }^{10}$ In an experimental study, Munro ${ }^{16}$ demonstrated that the intervertebral disc together with the anterior and posterior longitudinal ligaments provide significant cervical spine stability. By removing two or all of these three components, ACD impairs this stability. Interbody fusion is performed to restore the height of the disc space and intervertebral foramen, restrict mobility, and prevent collapse of the disc space with subsequent kyphotic deformity. ${ }^{24}$ Fusion may also lead to absorption of osteophytes $^{5}$ (Fig. 2).

There is no universally accepted method for ACDF. In a comparative study between patients treated with simple ACD and those treated with tricortical autograft-associated ACDF, Watters and Levinthal ${ }^{23}$ concluded that the ideal procedure would be ACDF involving a biomaterial that is minimally expensive and consistently promotes successful arthrodesis. The ideal implantation material after ACDF has not yet been found. In the last few years there has been a growing trend in the application of cage devices for spinal interbody fusion, both lumbar and cervical. Different types of cages with different shapes (cylindrical, conical, or cuboidal) are commercially available, most made from titanium alloy. Some cages are designed for reconstruction after corpectomy, whereas others are suitable for replacement of the intervertebral disc. ${ }^{15}$

In our review of the literature we found only one publication in which the authors reported clinical results of CFC-assisted ACDF. ${ }^{3}$ In that report, Brooke, et al., ${ }^{3}$ described the treatment of 19 patients in whom ACDF was performed using CFCs packed with cancellous bone graft obtained from the iliac crest. Solid arthrodesis was achieved in all their patients without cage-related complications. By using bone fragments from osteophytes at the surgically targeted disc level for grafting, we eliminated the potential risks of autogenic graft harvest. Isu, et al., ${ }^{12}$ reported a method of noninstrumented ACDF in which bone graft is obtained from the VBs. Unlike these authors, we use only bone from the osteophytes that have to be removed for adequate decompression, and we do not harvest any other bone from the VBs. The observation that new bone grows from the adjacent endplates, rather than from the bone graft, raises the possibility that a bone graft may be unnecessary for cage-assisted ACDF; however, further experimental and clinical studies are required to elaborate this issue.

In contrast to the paucity of clinical reports, several studies have been published on biomechanical characteristics of the CFC. In an experimental study, Cunningham, et al., ${ }^{7}$ showed that the stiffness of the CFC in axial compression was 10-fold greater than that of autologous fresh-frozen or freeze-dried iliac bone graft. Shono, et al., ${ }^{20}$ conducted a biomechanical study in which they analyzed fresh-frozen cervical spines that were obtained from calves and subjected to anterior decompression and reconstruction with polymethylmethacrylate, iliac autograft, or carbon fiber-reinforced cage. The CFC was most rigid in flexion-extension and also provided good stiffness in axial compression and rotation (more so than iliac graft but less than polymethylmethacrylate). The authors concluded that the CFC had great mechanical potential in anterior cervical reconstruction. Wilke, et al., ${ }^{24}$ examined subsidence as a result of postoperative movements in cadaveric cervical spines treated with bone cement and different fusion cages (BAK, WING, and Acromed carbon cages). They found that the CFC had better resistance to subsidence than the WING and BAK cages. All three cages had higher subsidence than bone cement. The subsiding BAK cage partially supported stability whereas the subsiding WING and carbon cages did not. These authors also showed that postoperative simulated neck movements increased the range of motion in all four groups. After cyclic loading, the range of motion tended to be smaller in the specimens treated with carbon cage or with bone cement than in the specimens in which the WING and BAK cages were used. ${ }^{13}$ Bartels, et al., ${ }^{1}$ studied the results fixated in 13 patients treated by CFC-assisted ACDF and reported that the height of the nerve root foramen increased after implantation of the cage and that this increase persisted after 1 year. In our study we found no evidence of significant subsidence in any of the patients during the follow-up period.

No CFC related complications were encountered in our study, nor in the two previously reported clinical studies., ${ }^{1,3}$ The only case of CFC-related failure was reported after lumbar interbody fusion. In that case, the broken cage was detected during reoperation after a prolonged period of infection. $^{22}$

Another controversial issue concerning ACDF is the need for anterior plating. Zdeblick and coworkers ${ }^{26}$ have demonstrated in an animal study that the use of a plate system did not significantly increase the rate of arthrodesis in three-level anterior cervical fusions. Shapiro ${ }^{18}$ and Shapiro, et al., ${ }^{19}$ reported achieving excellent results after performing $\mathrm{ACDF}$ in which they used cadaveric fibula and a locking plate compared with autogenic iliac graft. An anterior plate construct would increase the stiffness of the fixation and prevent extrusion of the interbody implant. Plating, however, is more costly, requires longer operative time, and may be associated with problems such as breakage or dislocation of the screws and perforation of adjacent structures. In addition, other questions such as the effect of the plate system on the risk for adjacent-segment morbidity remain unanswered. In our experience the use of the CFC for ACDF provided immediate and adequate stabilization as well as restoration of physiological lordosis in the surgically treated segment. There were no cases of cage displacement or breakage, and vertebral stability was maintained until solid fusion developed. We suggest that an anterior plate system should be added to reinforce the cage-assisted fusion only in cases of obvious instability such as traumatic disc herniation associated with either dislocation or ligamentous injury, and previous destabilizing surgery.

An important feature of the CFC is its radiolucency. Because it is not visualized on plain $\mathrm{x}$-ray films, better radiographic assessment of bone fusion is achieved. The position of the cage, however, can be determined by the radiopaque tantalum beads incorporated within it. The cage is also computerized tomography and MR imaging compatible and causes no significant artifacts that would impede the clarity of follow-up imaging (Fig. 4). 


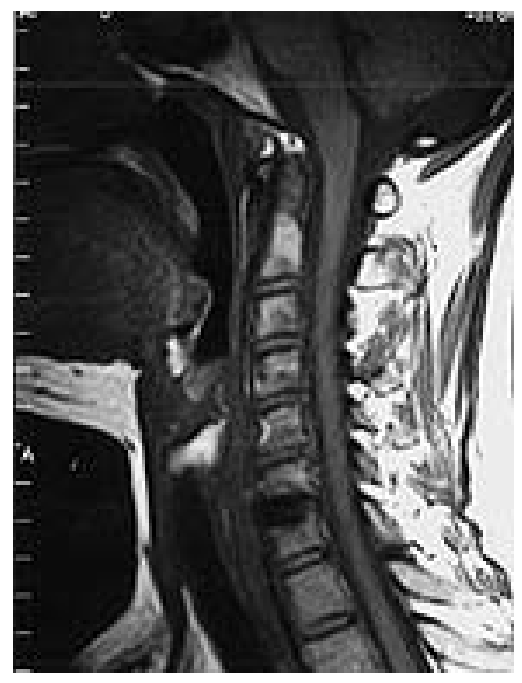

Fig. 4. Sagittal MR image obtained after ACDF in which a CFC was placed at C6-7, revealing adequate decompression of the spinal cord. The MR imaging-compatible cage is not visualized, and there are no significant artifacts.

Some features of cage-assisted cervical interbody fusion remain to be tested, such as the cost effectiveness of the device, as well as the long-term follow-up results of fusion compared with those obtained using other methods of cervical interbody fusion.

\section{CONCLUSIONS}

This paper summarizes our short-term experience with the CFC in interbody fusion after ACD. Analysis of our results suggests that this construct is safe, easy to use, and effective as an adjunct for interbody fusion after ACD. Additional studies, particularly those focused on longterm results, comparison of CFCs with different cervical cages or other substitutes, and cost effectiveness, are needed to establish the role of this device in cervical interbody fusion.

\section{Disclaimer}

The authors have no financial interest in the products or methodology described in this paper.

\section{References}

1. Bartels RHMA, Donk R, Van Dijk Azn R: Height of cervical foramina after anterior discectomy and implantation of a carbon fiber cage. J Neurosurg 95 (Suppl 1):40-42, 2001

2. Blakemore ME: Fractures at cancellous bone graft donor sites. Injury 14:519-522, 1983

3. Brooke NS, Rorke AW, King AT, et al: Preliminary experience of carbon fiber cage prostheses for treatment of cervical spine disorders. Br J Neurosurg 11:221-227, 1997

4. Caspar W, Pitzen T: Anterior cervical fusion and trapezoidal plate stabilization for re-do surgery. Surg Neurol 52:345-352, 1999

5. Cloward RB: Special considerations: bone as a spinal instrument, in Benzel EC (ed): Spinal Instrumentation. Park Ridge, IL: AANS, 1994, pp 185-210

6. Cowley SP, Anderson LD: Hernias through donor sites for iliacbone grafts. J Bone Joint Surg Am 65:1023-1025, 1983
7. Cunningham BW, Brantigan J, Shono Y, McAfee PC, Steffee A: Reconstruction of anterior spinal column defects utilizing a carbon fiber reinforced composite. Trans Orthop Res Soc 17: 168, 1992 (Reference unverified)

8. Escalas F, DeWald RL: Combined traumatic arteriovenous fistula and ureteral injury: a complication of iliac bone-grafting. J Bone Joint Surg Äm 59:270-271, 1977

9. Fathie K: Anterior cervical diskectomy and fusion with methyl methacrylate. Mt Sinai J Med 61:246-247, 1994

10. Goel VK, Clausen JD: Prediction of load sharing among spinal components of a C5-C6 motion segment using a finite element approach. Spine 23:684-691, 1998

11. Hafez RF, Crockard HA: Failure of osseous conduction with cervical interbody BOP graft. Br J Neurosurg 11:57-59, 1997

12. Isu T, Kamada K, Kobayashi N, et al: The surgical technique of anterior cervical fusion using bone grafts obtained from cervical vertebral bodies. J Neurosurg 80:16-19, 1994

13. Kettler A, Wilke HJ, Claes L: Effects of neck movements on stability and subsidence in cervical interbody fusion: an in vitro study. J Neurosurg 94 (Suppl 1):97-107, 2001

14. Kim P, Wakai S, Matsuo S, et al: Bisegmental cervical interbody fusion using hydroxyapatite implants: surgical results and long-term observation in 70 cases. J Neurosurg 88:21-27, 1998

15. McAfee PC: Interbody fusion cages in reconstructive operations on the spine. J Bone Joint Surg Am 81:859-880, 1999

16. Munro D: The factors that govern the stability of the spine. Paraplegia 3:219-228, 1966

17. Sawin PD, Traynelis VC, Menezes AH: A comparative analysis of fusion rates and donor-site morbidity of autogeneic rib and iliac crest bone grafts in posterior cervical fusions. J Neurosurg 88:255-265, 1998

18. Shapiro S: Banked fibula and the locking anterior cervical plate in anterior cervical fusions following cervical discectomy. $\mathbf{J}$ Neurosurg 84:161-165, 1996

19. Shapiro S, Connolly P, Donnaldson J, et al: Cadaveric fibula, locking plate, and allogeneic bone matrix for anterior cervical fusions after cervical discectomy for radiculopathy or myelopathy. J Neurosurg 95 (Suppl 1):43-50, 2001

20. Shono Y, McAfee PC, Cunningham BW, et al: A biomechanical analysis of decompression and reconstruction methods in the cervical spine. Emphasis on a carbon-fiber-composite cage. J Bone Joint Surg Am 75:1674-1684, 1993

21. Toth JM, An HS, Lim TH, et al: Evaluation of porous biphasic calcium phosphate ceramics for anterior cervical interbody fusion in a caprine model. Spine 20:2203-2210, 1995

22. Tullberg T: Failure of a carbon fiber implant. A case report. Spine 23:1804-1806, 1998

23. Watters WC III, Levinthal R: Anterior cervical discectomy with and without fusion. Results, complications, and long-term follow-up. Spine 19:2343-2347, 1994

24. Wilke HJ, Kettler A, Goetz C, et al: Subsidence resulting from simulated postoperative neck movements: an in vitro investigation with a new cervical fusion cage. Spine 25:2762-2770, 2000

25. Younger EM, Chapman MW: Morbidity at bone graft sites. J Orthop Trauma 3:192-195, 1989

26. Zdeblick TA, Cooke ME, Wilson D, et al: Anterior cervical discectomy, fusion and plating. A comparative animal study. Spine 18:1974-1983, 1993

27. Zdeblick TA, Ducker TB: The use of freeze-dried allograft bone for anterior cervical fusions. Spine 16:726-729, 1991

Manuscript received November 5, 2001.

Accepted in final form December 19, 2001.

Address reprint requests to: Khalil Salame, M.D., Department of Neurosurgery, Tel Aviv Sourasky Medical Center, 6 Weizmann Street, Tel Aviv, 64239, Israel. email: salame@ tasmc.health.gov.il. 